\title{
2 Einführung von Qualitätsmanagement in der psychotherapeutischen Praxis
}

Julia Bellabarba

Die Einführung von QM in der psychotherapeutischen Einzelpraxis stellt erfahrungsgemäß eine Herausforderung dar. Das Projekt QM wirkt wie ein Berg an nicht anwendbaren, teilweise unverständlichen Vorgaben, und es fällt schwer, ohne Anleitung und fachspezifische Einführung und ohne Diskussionsmöglichkeiten im Team, den Anfang zu machen. In der Regel ist es daher sinnvoll, sich mit aufgeschlossenen Kollegen zusammenzuschließen und die gewinnbringenden Aspekte einer QM Ausrichtung gemeinsam zu diskutieren und umzusetzen. Wir möchten hier, sehr pragmatisch, vier Vorgehensweisen vorschlagen, die den Einstieg in das QM für Psychotherapeuten in Einzelpraxis oder Praxisgemeinschaften erleichtern.

\subsection{Die Selbstbewertung der Praxis anhand der GBA Vorgaben als Ausgangspunkt}

Bei dieser Vorgehensweise wird der Selbstbewertungsbogen der KBV, der hier in modifizierter Form für Psychotherapeuten wiedergeben wird, genutzt, um festzustellen, welche Aspekte der QM-Richtlinie des GBA in der Praxis noch nicht verwirklicht wurden. Daraus ergeben sich konkrete Anhaltspunkte, welche Aspekte der eigenen Arbeit optimierbar sind. Ein realistischer Zeitplan mit fester Terminierung der einzelnen Abschnitte ist notwendig, am besten im Austausch mit Kollegen, um die Verbindlichkeit im „Ein-Personen-Team“ zu erhöhen.

- Ich integriere aktuelle fachliche und wissenschaftlich anerkannte Standards in den Behandlungsalltag.

- Ich berücksichtige bei der Versorgung meiner Patienten Leitlinien. 
- Ich kümmere mich um die Sicherheit der Patienten, z. B. Erläuterungen zu einer möglichen Verschlechterung der Symptomatik im Behandlungsverlauf, Risikomanagement im Umgang mit Suizidalität.

- Patienten werden beraten und können bei Entscheidungen mitwirken. Beratungen können zu Erkrankungen, Änderung konkreter Verhaltensweisen und präventiven Themen durchgeführt werden. Psychotherapeutischer Behandlung sollte immer ein (dokumentiertes) Entscheidungsgespräch vorangehen, in dem der Patient konkret auf Alternativen hingewiesen wird (Hinweise auf andere Einrichtungen, Selbsthilfegruppen, andere mögliche Psychotherapieformen). Siehe auch Exkurs zur Therapie-Einwilligung am Ende des Abschnitts.

- Patienten werden über präventive, diagnostische und therapeutische Maßnahmen informiert.

- In meiner Praxis werden die wichtigsten Behandlungsabläufe strukturiert. Dies bedeutet, dass der Ablaufvon wichtigen Behandlungen klarfestgelegt ist. In diesen Abläufen sollten neben der psychotherapeutischen Vorgehensweise auch dazugehörige praxisorganisatorische Elemente beschrieben sein.

- Die externe Kommunikation der Praxis ist geregelt.

- Das Informationsmanagement in der Praxis ist festgelegt. Hierzu gehört die Weitergabe von (patientenbezogenen) Informationen außerhalb der Praxis sowie der strukturierte und regelmäßige Austausch wichtiger Informationen im Rahmen der kollegialen Intervision und mit den Patienten (wichtig dabei zu berücksichtigen: ärztl. Schweigepflicht und Bundesdatenschutzgesetz!).

- Die Praxis kooperiert mit anderen Versorgungsbereichen und hat die Nahtstellen zu diesen beschrieben.

- Ich habe für die Praxis konkrete Qualitätsziele festgelegt.

- Ich überprüfe systematisch, ob meine Ziele erreicht wurden.

- Ich führe Patientenbefragungen in der Praxis durch.

- Der Umgang mit Beschwerden in der Praxis ist geregelt.

- In der Praxis werden Checklisten verwendet. Checklisten können bspw. für immer wiederkehrende Arbeiten, zum Kontrollieren von Bereichen oder zur Vorbereitung von Maßnahmen eingesetzt werden.

- In meiner Praxis habe ich festgelegt, wie ich mit Fehlern und Beinahefehlern umgehe. Fehler oder Beinahefehler können of bereits im Vorfeld vermieden werden. Geschehene Fehler können analysiert und genutzt werden, um Verbesserungen umzusetzen.

- Für die Versorgung von Notfallpatienten gibt es in meiner Praxis ein festgelegtes Notfallmanagement.

- Behandlungsverläufe und Patientenberatungen werden in meiner Praxis dokumentiert.

- In meiner Praxis werden qualitätsbezogene Dokumentationen, insbesondere die Dokumentation der Qualitätsziele der Praxis und der ergriffenen Umsetzungsmaßnahmen und die Dokumentation der systematischen Überprüfung der Zielerreichung durchgeführt. 


\subsection{Einführung von QM auf der Grundlage eines QM-Systems}

Eine detaillierte Beschreibung der vorliegenden QM-Systeme für die ambulante Patientenversorgung finden Sie in Kapitel I.4 und in Anhang 3. Die Entscheidung für ein QM-System sollte mit der Frage verbunden sein, ob die Praxis zertifiziert werden soll oder nicht. QEP $^{\circledR}$ liegt in der Version der Deutschen Psychotherapeutenvereinigung mit Musterdokumenten vor, die allerdings nur für psychologische Psychotherapeuten geeignet sind, da ärztliche Leistungen (wie zum Beispiel Verordnung von Medikamenten) nicht berücksichtigt werden. Die Vereinigung Analytischer Kinder- und Jugendlichenpsychotherapeuten hat ebenfalls Musterdokumente entwickelt, die der $\mathrm{QEP}^{\circledR}$ Systematik entsprechen (leider vergriffen). Im Juni 2009 soll ein eigenes $\mathrm{QEP}^{\circledR} \mathrm{Ma}-$ nual der KBV für Psychotherapeuten auf den Markt kommen. Weitere zertifizierbare QM-Systeme, die auch auf psychotherapeutische Praxen übertragbar sind, (aber keine PT-spezifischen Musterdokumente enthalten) sind KTQ, EPA, KVWL/qu.no und ISO. Der Vorteil, das eigene QM-Projekt durch ein System zu strukturieren, liegt auf der Hand: das System führt den Benutzer Schritt für Schritt durch die einzelnen Arbeits- und Organisationsbereiche, die im Rahmen der GBA-Richtlinie berücksichtigt werden müssen (und, je nach System, auch etwas darüber hinaus). Der Praxisinhaber hat also bei der Umsetzung der genannten Systeme die Gewähr, die gesetzlichen Vorgaben mit Sicherheit zu erfüllen.

\subsection{Einführung von QM nach dem Lustprinzip}

Um die anfängliche Motivation zu erhöhen, wäre es auch denkbar, mit konkreten QM-Projekten zu starten, die eine unmittelbare Arbeitserleichterung für den Praxisinhaber mit sich bringen. Zum Beispiel:

- regelmäßige Erfassung der Bewilligungs- bzw. Ablehnungsquote für Therapiegutachten

- Einsatz von Fragebögen zur Anamnese zur Vorbereitung auf die Gutachtenerstellung

- Verbesserung der internen Kommunikation in einer Praxisgemeinschaft

- Verbesserung der Patienteninformation (beispielsweise in Form von Flyern, Mappen, Websites)

- Vereinfachung des Briefwechsels mit Krankenkassen anhand vorgefertigter Textbausteine

- Beschleunigung des Weges zum Gutachtenbericht

- computergestützte Terminierung der Verlängerungsanträge

- Erstellung von Informationsmaterial für Patienten und Bezugspersonen

- Umstieg auf PC-Abrechnung

- Organisation einer interkollegialen Beratungsgruppe zum Thema QM 
- Erstellung einer Checkliste, die dem Therapeuten die Information der Patienten im Rahmen der Probatorik erleichtern (s. Tab. 8)

- Erstellung von Checklisten, die dem Therapeuten die Information der Patienten bei Beendigung der Therapie erleichtern (s. Tab. 9)

Tab. 8 Checkliste für Psychotherapeuten: Informationen im Rahmen der Probatorik

\section{Information der Patienten im Rahmen der Probatorik:}

Vorschläge für eine Checkliste für Psychotherapeuten

Informationen zur Entscheidung über die Therapieaufnahme

Information über das Setting, die Dauer einer Therapie, die Kosten und die Finanzierung

Antrags-, Gutachter- und Abrechnungsverfahren (inklusive Einlesen der Chipkarte)

Zusicherung der Verschwiegenheit

Hinweis auf mögliche Ausnahmesituationen (pflichtgemäße Aufklärung nach $§ 11$ BO PKN)

Informationen über Maßnahmen, die die Zustimmung des Patienten erfordern (wie Mitschnitt der Sitzungen auf Datenträgern oder Weitergabe von Daten an Dritte)

Entscheidung zur gemeinsamen therapeutischen Arbeit

Erläuterung alternativer Therapie- und Beratungsmöglichkeiten (Therapieplatzvermittlungsstellen, Therapeutenlisten der Krankenkassen)? Behandlungsalternativen (stationäre Therapie)?

ggf. Vereinbarung über weitere diagnostische bzw. probatorische Sitzungen

erste Hypothesen über die Problemlage des Patienten

Tab. 9 Checkliste für Psychotherapeuten: Informationen bei Therapieende

Information der Patienten bei Abschluss der Behandlung:

Vorschläge für eine Checkliste für Psychotherapeuten

Zeitpunkt und Modus des Abschlusses der Behandlung werden i.d. R. von Patient und Therapeut gemeinsam festgelegt.

Gemeinsame Planung der Abschlussphase:

Beendigung mit der letzten Stunde des Therapiekontingents im üblichen Zeittakt

Reduzierung der Sitzungsfrequenzen

zeitlich festgelegte Therapiepause bzw. Erprobungsphasen im Rahmen bestimmter Therapieziele

ggf. Vereinbarung über ein offenes „Sitzungskontingent“, das im Bedarfsfall vom Patienten genutzt werden kann

Abschlusssitzung:

Benennung der Behandlungsergebnisse aus Therapeuten- und Patientenperspektive

Thematisierung der Erfolge und Schwierigkeiten

Auswertung der Veränderungsmessung

ggf. Behandlungsalternativen bzw. weiterführende Behandlungsmöglichkeiten aufzeigen und/ oder vermitteln

Vereinbarung über Kontaktmöglichkeiten nach Beendigung der Therapie 


\subsection{Einführung von QM nach dem Pflichtprinzip}

- Frage: Muss ich einen rollstuhlgerechten Zugang zu meiner Praxis haben? Antwort: Nein.

- Frage: Brauche ich getrennte Damen- und Herrentoiletten? Antwort: Nein.

- Frage: Brauche ich einen Feuerlöscher in der Praxis? Antwort: Ja, wenn Sie Mitarbeiter beschäftigen und auch sonst empfehlenswert.

Nicht immer ist in der psychotherapeutischen Praxis gegenwärtig, welche Gesetze, Verordnungen und Vorgaben für den Inhaber verpflichtend sind. Es macht daher Sinn, Qualitätsmanagement in der Praxis einzuführen indem sich der Praxisinhaber als erstes fragt: Was muss ich umsetzen? Wie weit bin ich hinsichtlich der Erfüllung geltender Normen? Eine Übersicht über die derzeitig geltenden Normen/Gesetze/Richtlinien/Vorgaben, die für psychotherapeutische Praxen gelten, finden Sie in Anhang 1. Den meisten Therapeuten wird es wahrscheinlich widerstreben, sich nun die gesammelten Gesetzeswerke vorzunehmen, durchzulesen und auf Anwendung zu überprüfen. Wir stellen Ihnen daher eine Reihe rechtsrelevanter Themen und Fragen vor, die als Einstieg sinnvoll genutzt werden können.

(Sie finden eine stets aktuelle Liste der Rechtsnormen, die für Praxen gelten, über folgenden Link: www.kvhh.net/public/media/Dokumente/unser_ service_fuer_sie/aerzte__psychotherapeuten/qualitaetsmanagement/qm_ rechtsvorschriften_mit_linkliste.pdf).

Vorgaben der Berufsgenossenschaft: Die Anmeldung des Praxisinhabers bei der Berufsgenossenschaft für Gesundheit und Wohlfahrtspflege (www.bgw-online.de) ist in jedem Fall erforderlich, die BGW ist auch für sämtliche Fragen zur Sicherheit und Hygiene in der Praxis zuständig (solche Fragen, wie die am Anfang des Kapitels erwähnten). Insgesamt sind es nur sehr wenige, durchaus plausible Vorgaben, die in dieser Hinsicht dem Psychotherapeuten gemacht werden, da die psychotherapeutische Praxis kaum Gefahren in Hinblick auf Ansteckung, Kontaminierung, Verstrahlung oder Vergiftung aufweist. Zum Thema Hygiene: In der psychotherapeutischen Praxis sind keine „besonderen“ Hygienevorschriften zu beachten, es reicht die normale „Haushaltshygiene“. Sollte eine PT-Praxis Patienten betreuen, die ein Ansteckungsrisiko im normalen zwischenmenschlichen Kontakt darstellen (wie z. B. Tuberkulosekranke), sind die Vorgaben der Berufsgenossenschaft anzuwenden.

Haftung: Risiken, die die Sicherheit der Patienten betreffen und die als vermeidbar und kontrollierbar einzuordnen sind. Dieser Aspekt betrifft also das Risikomanagement einer Praxis. Es „hat zum Ziel, aktiv nach Schadensursachen und Risikofeldern zu suchen, um Haftungsfälle präventiv zu vermeiden, was - umgekehrt - positive Behandlungsqualität impliziert“ (Bock 2009, S. 5). In der psychotherapeutischen Praxis bedeutet das konkret, sich Regelungen für Notfälle zuzulegen (Suizidalität, psychotische Dekompensation, Fremdgefährdung). Dazu gehören schriftliche Vereinbarungen mit gefährdeten Patienten über die Erreichbarkeit 
des Therapeuten. Ebenso die Dokumentation der (telefonischen) Kontaktversuche des Therapeuten bei Anruf oder Anrufbeantworternachricht, auch bei Patienten, die nicht in Behandlung sind. Ganz banal gehören auch Aspekte der Sicherheit in den Praxisräumen dazu: Besonders bei der Behandlung von Kindern sind potenzielle Gefährdungen zu beheben (offene Fenster, Steckdosen, spitze Gegenstände). Eine weitere Überlegung könnte die Verkehrsicherheit der Patienten betreffen: Wie sicher schätzt der Therapeut den Patienten ein, nach einer emotional aufwühlenden Therapiesitzung?

Therapie-Einwilligung durch den Patienten: Aufgrund tradierter Vorgaben wird jede ärztliche (auch psychotherapeutische) Behandlung a priori als „Körperverletzung“ definiert (auch wenn Indikationsstellung und Behandlungsdurchführung völlig korrekt sind). Es besteht also eine (zumindest juristische) Äquivalenz sämtlicher Interventionsformen innerhalb des medizinischen Systems, das die psychologisch-psychotherapeutische einschließt. Diese Grundannahme mag realitätsfern wirken - gerade im Zusammenhang mit einer psychotherapeutischen Behandlung, die ja, zumindest in körperlicher Hinsicht, absolut nicht invasiv ist. Wichtig ist es trotzdem, gerade für psychologische Psychotherapeuten, dies zur Kenntnis zu nehmen. Aus diesem Verständnis heraus ergibt sich nämlich die rechtliche Forderung nach eindeutiger, dokumentierter Einwilligung des Patienten, und zwar auf der Grundlage adäquater Aufklärung durch den Therapeuten zu Risiken der Psychotherapie und Behandlungsalternativen. Dementsprechend besteht bei der (psychotherapeutischen) Behandlung von Kindern die Verpflichtung, eine Zustimmung zur Behandlung von den (sorgeberechtigten) Eltern schriftlich einzuholen (im Fall getrennt lebender Eltern, bedeutet das: beider sorgeberechtigter Eltern.)

Datenschutzgesetz und Schweigepflicht: Umsetzung der Vorgaben des Bundesdatenschutzgesetzes und der Berufsordnung bezüglich der Schweigepflicht. Der Verstoß gegen das ärztliche/psychologische Berufsgeheimnis begründet nicht nur eine Berufspflichtverletzung, sondern hat auch eine strafrechtliche Bedeutung. Vereinfacht, flapsig formuliert: Durch diese beiden Regelwerke ist festgelegt, dass alles verboten ist, was nicht erlaubt ist. Das heißt: jede Weitergabe und Mitteilung von Patientendaten muss vom Patienten (schriftlich lt. Datenschutzgesetz) autorisiert werden! Der Psychotherapeut sollte besondere Sorgfalt darauf verwenden, die Datenweitergabe an die Krankenkassen (GKV und PKV), die Sozial- und Jugendämter, die Schulen und Lehrer der Patienten stringent im Sinne dieser Vorgabe zu organisieren. Auch wird häufig missachtet, dass die Verschwiegenheitspflicht gegenüber jedermann besteht, so auch:

- gegenüber den eigenen Familienangehörigen,

- gegenüber Familienangehörigen des Patienten,

- gegenüber anderen Ärzten/Psychologen,

- gegenüber demjenigen, der von der betreffenden Tatsache bereits Kenntnis erlangt hat und

- dass die Verschwiegenheit auch nach dem Tod des Patienten fortbesteht. 
Sollten Andere im Rahmen ihrer Tätigkeit für die Praxis über Patienten Informationen erhalten (beispielsweise als Reinigungskraft, EDV-Spezialist, Schreibdienst) gilt diese Verpflichtung ebenso stringent und sollte schriftlich durch eine Verschwiegenheitserklärung dokumentiert werden.

Dokumentationspflicht:als Nebenpflicht im Rahmen des Behandlungsvertrags. Die Pflicht, Diagnose und therapeutischen Prozess zu dokumentieren, dient in erster Linie der Gedächtnisstütze des Therapeuten, in zweiter Linie der Nachvollziehbarkeit im Rahmen einer Plausibilitätsprüfung durch die KV und zuletzt auch der Beweisbarkeit im Rahmen haftungs- und strafrechtlicher Auseinandersetzungen. Zusammenfassen lässt sich diese Verpflichtung folgendermaßen: Die Dokumentation der Patientenkontakte kann schriftlich oder/und digital erfolgen (für die elektronische Datenspeicherung muss der Patient sein Einverständnis erteilen). Empfehlenswert ist eine Dokumentationsform, die zwischen „Persönlichen Aufzeichnungen des Therapeuten“ und Dokumentation der Behandlung differenziert. Nur für letzteres gilt das Recht des Patienten auf Akteneinsichtnahme. Die Dokumentation des Behandlungsverlaufs hat vom ersten Kontakt an lückenlos zu erfolgen und sollte zeitnah (innerhalb von 24 Stunden) erfolgen, Notfälle oder besondere Vorkommnisse müssen sofort festgehalten werden. Die Patientenakte muss mindestens folgende Angaben enthalten:

- ICD-1o Diagnose (und differentialdiagnostische Überlegungen)

- biographische Anamnese

- Fallkonzeptualisierung, Indikationsstellung und Therapieplan

- Verlaufsdokumentation

Arbeitsstättenverordnung: dient der Sicherheit und dem Gesundheitsschutz der Beschäftigten. In einer psychotherapeutischen Praxis bestehen nur sehr wenige „Gefahren“ für Beschäftigte (und die meisten Psychotherapeuten haben keine Mitarbeiter). Überlegungen in dieser Hinsicht sollten also dazu beitragen, das (geringe) Gefährdungspotenzial realistisch einzuschätzen: Brandschutz (Feuerlöscher), Gesundheitsschutz am Bildschirm, Sturzgefahren wie Kabel, Schwellen, usw. Darüber hinaus steht es natürlich jedem Psychotherapeuten frei, für sich selbst ergonomisch optimierte Arbeitsbedingungen zu schaffen.

\section{Literatur}

Behnsen E, Bell K, Best D et al. (Hrsg.): Management Handbuch für die psychotherapeutische Praxis (ständig aktualisierte Loseblattsammlung). Heidelberg: Psychotherapeutenverlag Stand 37. Aktualisierung Dezember 2008

Bock R-W: Recht für Krankenhaus und Arztpraxis. Berlin: Medizinisch Wissenschaftliche Verlagsgesellschaft 2009

Herzog T, Stein B, Wirsching M: Qualitätsmanagement in Psychotherapie und Psychosomatik. Stuttgart: Thieme 2000

Lairaiter A-R, Vogel H (Hrsg.): Qualitätssicherung in der Psychotherapie und psychosozialen Versorgung. Tübingen: DGVT-Verlag 1998 
Laireiter A-R, Vogel H (Hrsg.): Qualitätssicherung in der Psychotherapie. Psychotherapiepraxis. Psychotherapie, 6. Jahrg 2001; 6: 1, 21

Lambert MJ (Hrsg.): Bergin and Garfield's Handbook of Psychotherapy and Behavior Change. 5. Aufl. New York: John Wiley \& Sons 2004

Liebold R, Zalewski T: Handbuch Psychotherapie in der vertragsärzlichen Versorgung (Grundwerk mit 26. Lieferung) (Ringeinband). Sankt Augustin: Asgard Verlag 2006

Linster H-W, Härter M, Stieglitz R-D (Hrsg.): Qualitätsmanagement in Psychotherapie und Beratung. Grundlagen - Methoden - Anwendung. Göttingen: Hogrefe 2004

Nathan P, Gorman I (Hrsg.): A guide to treatments that work. 2. Aufl. New York: Oxford University Press 2002

Piechotta B, Müller U: Qualitätssicherung für niedergelassene analytische Psychotherapeuten (QNAP). Evaluation eines QS-Projektes von niedergelassenen Psychoanalytikern in NRW 2001-2004. Forum der Psychoanalyse 2008; 24: 382-394

Piechotta B: PsyQM. Qualitätsmanagement für psychotherapeutische Praxen. Berlin, Heidelberg: Springer 2008

Piechotta B: Stein der Weisen oder Stein des Sisyphos? Qualitätsmanagement und Zertifizierung für Praxen von Psychoanalytikern. Forum der Psychoanalyse 2003; 19: 129-148

Stieglitz R-D, Härter M, Linster HW (Hrsg.): Qualitätsmanagement in der Psychotherapie. Grundlagen, Methoden und Anwendungen. Göttingen, Bern, Toronto, Seattle: Hogrefe 2003

\section{Links}

QM-Richtlinien der Bundes PTK: www.bptk.de/aktuelles/news/94287.html

www.ivt-hessen.de

www.kbv.de/themen/qualitaetsmanagement

www.deutschepsychotherapeutenvereinigung.de

www.g-ba.de

www.kbv.de/themen/6102.html

www.bgw-online.de

www.kbv.de/qep/11625.html

www.kvb.de/servlet/PB/show/1116432/QM-Richtlinienbroschuere_4_2008.pdf

Aktuelle Liste der Rechtsnormen, die für Praxen gelten, über folgenden Link: www.kvhh.net/public/media/ Dokumente/unser_service_fuer_sie/aerzte__psychotherapeuten/qualitaetsmanagement/qm_ rechtsvorschriften_mit_linkliste.pdf 